\section{Imaging the Sublimation Dynamics of Colloidal Crystallites}

\author{
J. R. Savage, ${ }^{1}$ D. W. Blair, ${ }^{1}$ A. J. Levine, ${ }^{2}$ R. A. Guyer, ${ }^{1}$ A. D. Dinsmore ${ }^{1}$
}

We studied the kinetics of sublimating crystals with single-particle resolution by experiments with colloidal spheres and by computer simulations. A short-range attraction between spheres led to crystallites one to three layers thick. The spheres were tracked with optical microscopy while the attraction was reduced and the crystals sublimated. Large crystallites sublimated by escape of particles from the perimeter. The rate of shrinkage was greatly enhanced, however, when the size decreased to less than 20 to 50 particles, depending on the location in the phase diagram. At this size, the crystallites transformed into a dense amorphous structure, which rapidly vaporized. The enhancement of kinetics by metastable or unstable phases may play a major role in the melting, freezing, and annealing of crystals.

$\mathrm{T}$ he process by which crystals melt into a liquid or sublimate into a gas are of considerable fundamental and practical importance. Even in model systems whose equilibrium configurations are known, the kinetics of phase transitions have proved difficult to understand and predict (1-4). In the case of nucleation of a crystalline phase from a fluid, the kinetics are slowed by the energy cost of forming an interface between the new phase and the old one, as described by classical nucleation theory. Complicating the process, thermodynamically metastable or unstable phases enhance the nucleation rate by lowering the free-energy barrier that must be overcome to reach equilibrium $(5-10)$. In studies of the reverse process - melting or sublimationtwo different mechanisms have been identified. Three-dimensional crystallites that have stabilized surfaces melt from within through mechanical or Born melting $(11,12)$ once the shear modulus is reduced to nearly zero (11-14). In this case, melting might be viewed as nucleation and growth of the fluid phase within the solid $(11,12,14)$. In two dimensions, large crystallites melt by a two-step process owing to the intervention of the hexatic phase, which appears in equilibrium $(3,15-19)$. In many practical cases, however, crystallites are of finite size. Melting or sublimation then take place at the surface (or perimeter) while the interior retains its crystalline order $(2,3)$. Thus, in experiments with real systems of melting or sublimating crystallites, the melting process is dominated by the surface (20). As crystallites shrink in size, one might expect the surface to play a continually greater role in the kinetics. Owing to the difficulty of studying small crystallites with single-particle resolution, however, this has remained an open question. Experiments with colloidal crystals at very low ionic strength have shown unexpectedly slow melting, but this has been attributed to a longrange attraction (21). We found that crystallites sublimate at a steady rate from their perimeter until they reach a characteristic size, after which they very rapidly vaporize.

${ }^{1}$ Department of Physics, University of Massachusetts Amherst, Hasbrouck Lab 411, 666 North Pleasant Street, Amherst, MA 01003, USA. ${ }^{2}$ Department of Chemistry and Biochemistry and California Nanosystems Institute, University of California, Los Angeles, CA 90095, USA.
Images of a typical colloidal crystallite while it sublimates into a low-density gas phase are shown in Fig. 1. The video images show colloidal spheres in water containing surfactant micelles (not visible). The micelles induce an attractive potential between spheres by the depletion, or excluded-volume, mechanism (22). The range of the depletion potential is determined by the diameter of the micelles, which is approximately 0.3 to $1.4 \%$ of the sphere diameter. The key to our experiment is that the size and concentration of the micelles - and hence the strength of attraction - are strong functions of temperature, $T$. Using the nonionic surfactant hexaethylene glycol monododecyl ether $\left(\mathrm{C}_{12} \mathrm{E}_{6}\right)$, we obtained crystallites of the colloidal spheres coexisting with a gas phase at $28^{\circ} \mathrm{C}$. These crystallites form at a flat glass surface owing to depletion attraction to the surface (23); most crystallites contain only a single layer of spheres. Cooling the sample to $27^{\circ} \mathrm{C}$ weakens the depletion attraction (22) and the crystallites sublimate (Fig. 1). During this transition, almost all of the spheres remain on the surface and diffuse in two dimensions. The images clearly indicate a two-stage process, in which large crystallites shrink by departure of particles from the perimeter monolayer at a rate limited by breaking of bonds (Fig. 1, A to C, time $\Delta t=100 \mathrm{~s}$ between images), whereas smaller clusters melt much more rapidly (Fig. $1, \mathrm{D}$ to $\mathrm{F}, \Delta t=15 \mathrm{~s}$ ).

To quantify the kinetics, Fig. 2 shows the cluster size $N(t)$ for several crystallites. Figure 2A shows data for three colloidal crystallites formed in the presence of $\mathrm{C}_{12} \mathrm{E}_{6}$ at $28^{\circ} \mathrm{C}$ and then cooled to $27^{\circ} \mathrm{C}$, similar to that in Fig. 1 . Here, $N$ is defined as the number of particles that are connected in a contiguous cluster by depletion bonds, which are defined by their separation (22). This definition of cluster is independent of the degree of crystalline order. For large clusters or early time, the rate of sublimation varies among crystallites, perhaps owing to variations in their shape. When $N$ falls below about 30 , however, the clusters are more isotropic in shape and in each case the rate markedly increases. We discerned no dependence of the final shrinkage rate on the cluster's history.

The results shown in Figs. 1 and 2A are robust; we observed similar results in other experiments and in computer simulations. Figure 2B shows data for four colloidal crystals formed in the presence of micelles of an anionic surfactant, sodium dodecyl sulfate (SDS), at room temperature. Two of these melted at $30^{\circ}$ and two melted at $65^{\circ} \mathrm{C}$; unlike the $\mathrm{C}_{12} \mathrm{E}_{6}$, the SDS micelles shrink with increasing $T$ (22). Figure $\mathrm{S} 1$ shows $N(t)$ for all seven crystallites that we studied at $65^{\circ} \mathrm{C}$. The typical rate of crystallite shrinkage for $N>30$ increases with $T$ by an order of magnitude, yet all samples show the break in the slope at $N \sim 20$ to 30. Figure $2 \mathrm{C}$ shows two crystallites melted in a Brownian dynamics computer simulation (22) of a quasi-two-dimensional system. The attractive pair potential approximated the depletion potential with a range equal to $10 \%$ of the particle diameter. These data adhered to the same enhanced kinetics at small $N(\approx 50$ in simulation), which excludes hydrodynamic flow as an explanation of our experimental results. Experimental data for colloidal crystals of two to three layers in thickness (fig. S2) also showed enhanced kinetics at small size, showing that the cross-over was not a strictly two-dimensional effect. All of these melting curves clearly showed a tendency to sublimate at a steady rate until $N$ reached a characteristic size. In crystallites below this size, the melting rate was greatly enhanced.

If the melting rate were limited by the rate of thermally breaking bonds at the perimeter, then $d N / d t \propto N^{1 / 2}$ and the melting rate should slow down rather than speed up. Alternatively, classical nucleation theory predicts a critical size for a growing
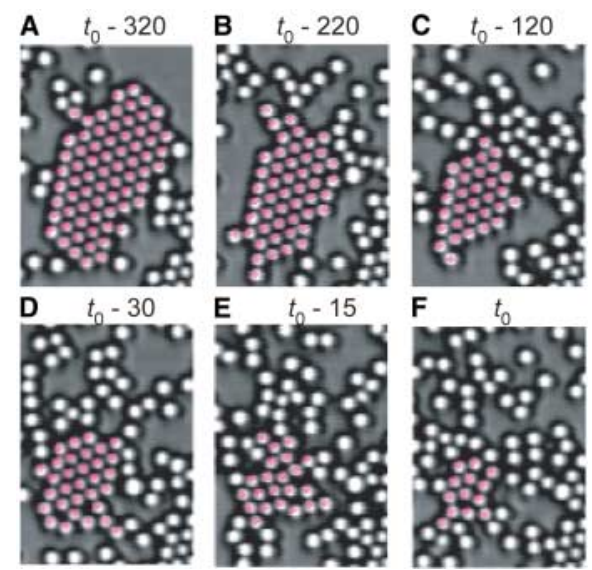

Fig. 1. Images of a crystallite of $0.7-\mu$ m-radius polystyrene spheres during sublimation. The purple dots indicate particles identified by the cluster algorithm. Images were obtained using an inverted optical microscope $(63 \times$ magnification, 1.3 numerical aperture) and a monochrome video camera. Times (in seconds) are measured relative to $t_{0}$, when the crystallites were defined as having vanished. The images indicate a relatively slow rate initially $[\Delta t=100 \mathrm{~s}$ (A to C)] and an enhanced rate near the end $[\Delta t=15 \mathrm{~s}$ ( $\mathbf{D}$ to $\mathbf{F})]$. The sample was heated from above and below with feedback-controlled resistive heaters. A sapphire plate was mounted to the top surface to suppress temperature gradients. The temperature had equilibrated and random fluctuations in temperature were approximately $0.1^{\circ} \mathrm{C}$. 


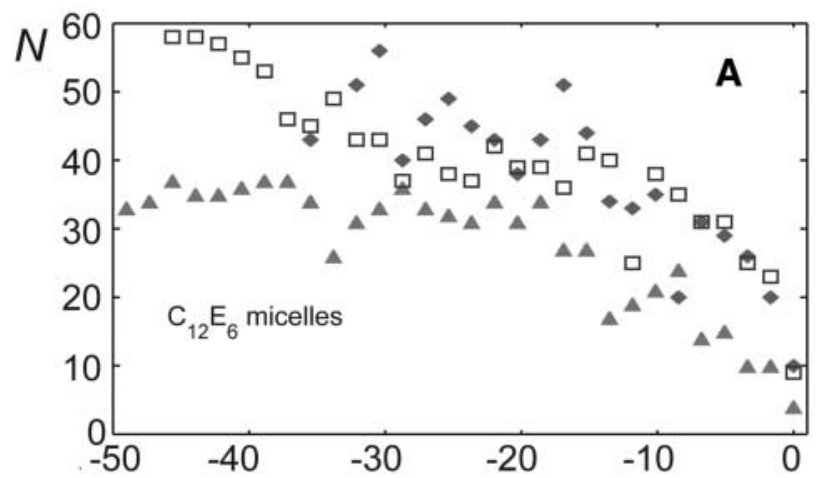

Fig. 2. Measured number of particles in individual clusters, $N$, versus time, $t$, normalized by the characteristic diffusion time $R^{2} I D$ (the time to diffuse a particle's diameter). For legibility, approximately every 40th data point is plotted. (A) Data from crystallites formed in the presence of $\mathrm{C}_{12} \mathrm{E}_{6}$ micelles with $30 \%$ area fraction. Squares, diamonds, and triangles indicate different clusters in the sample. The squares represent the sample shown in Fig. 1. (B) Data from crystallites formed in the presence of SDS micelles and heated to $30^{\circ} \mathrm{C}$ (triangles) and $65^{\circ} \mathrm{C}$ (diamonds). The area fractions were $16 \%$. (C) Data from two Brownian dynamics computer simulations with $24 \%$ area fraction. Triangles and diamonds indicate separate simulation runs.
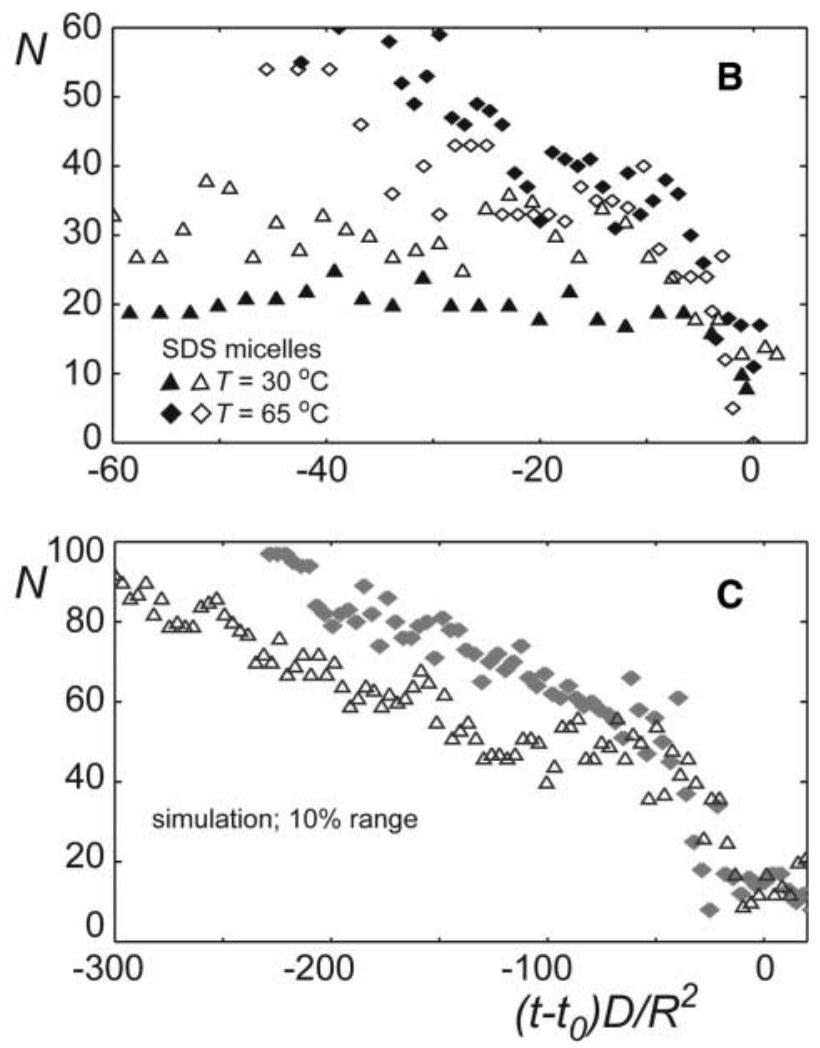

crystallite, which is reminiscent of the characteristic size reported here. However, in nucleation theory this size arises from a competition between the lower chemical potential of the crystal phase and its positive surface energy. During melting, the chemical potential is larger in the crystal, both terms are positive, and no critical size emerges. Instead, our analysis points to a third mechanism. We show that the cross-over in kinetics arises because of a twostep process: (i) The crystallite forms a dense amorphous phase, which is thermodynamically unstable, and (ii) it rapidly evaporates into the gas phase.

To distinguish crystalline from amorphous clusters, we measured the sixfold symmetry of bonds within the clusters using the bond-orientation order parameter, $\psi_{6}(15)$. For each particle (labeled $j)$ that has two or more bonds, $\psi_{6}(j) \equiv(1 / Z) \Sigma_{\mathrm{k}}$ $\exp \left[6 i \theta_{j k}\right]$, where $Z$ is the number of bonds with this particle, the sum is over all bonded neighbors $k$, and $\theta_{j k}$ is the angle between the $j-k$ bond and the $x$ axis. Computing the modulus squared, $\left|\psi_{6}\right|^{2}$ eliminates the arbitrary choice of axis. $\left|\psi_{6}\right|^{2}=1$ in a perfect hexagonal lattice, independent of the number of bonds. We found that the downturn in $N(t)$ is simultaneous with a drop in $\left|\psi_{6}\right|^{2}$ from between 0.8 and 1 to about 0.2 .

To focus on the effect of cluster size $N$ rather than time, we plotted $\left|\psi_{6}\right|^{2}$ av versus $N$, where the subscript "av" refers to an average over all clusters of size $N$ (Fig. 3A). For colloidal crystallites formed in the presence of $\mathrm{C}_{12} \mathrm{E}_{6}$ micelles and melting at $27^{\circ} \mathrm{C}$ (squares), $\left|\psi_{6}\right|^{2}{ }_{\text {av }}$ is notably reduced when $N$ is smaller than a cross-over value, $N_{\mathrm{x}} \approx 30$. A similar result was found in the presence of SDS micelles at $65^{\circ} \mathrm{C}$ (diamonds) and at $30^{\circ} \mathrm{C}$ (triangles), although in the latter case $N_{\mathrm{x}}$ is closer to 20 . In the computer simulation, with a range of attraction equal to $10 \%$ of the particle diameter, $N_{\mathrm{x}} 50$ (Fig. 3D).

Even more revealing of the loss of orientational order is the product of each particle's $\psi_{6}$ value with that of its neighbors, which we call $C_{6}(21)$ (Fig. $3 \mathrm{~B})$. In a dense fluid, particles may have a high degree of sixfold bond order; unlike in a crystal, however, the directions of the bonds vary from one region to another. Hence, the thermal average of the product of $\psi_{6}(i) \times \psi_{6}(j)^{*}$ vanishes when particles $i$ and $j$ are far apart. Indeed, long-range bond orientational $\left(\psi_{6}\right)$ order is a hallmark of twodimensional crystals, distinguishing them from hexatic and fluid phases, which exhibit quasilong-range (algebraically decaying) and short-range order, respectively (15). As expected, particles with $Z<6$ (which are near the perimeter) have lower values of $C_{6}$ than do their neighbors with $Z=6$ (Fig. 3B). As crystallites sublimate, an increasing fraction, $f$, of the particles have $Z<6$ (Fig. 3C). Notably, the crystallites do not lose order simply because $f$ increases, as might be expected if melting took place at a wetting layer. Instead, the particles with $Z<6$ (perimeter particles) themselves lose orientational order when $N<N_{\mathrm{x}}$. Particles with $Z=6$ and one layer in become very rare, but these also show reduced order (though the effect is small because packing six neighbors at short range requires nearly $60^{\circ}$ bond angles). Hence, small clusters have lower bond-orientational order throughout their area and should be characterized as amorphous.

These results point to the following mechanism for melting. Clusters larger than the cross-over size
$N_{\mathrm{x}}$ sublimate directly into the gas phase from the perimeter region. Here, particles at the perimeter typically have lower values of $\left|\psi_{6}\right|^{2}$ and $C_{6}$. In the simulations, we found that mean-square fluctuations in the bond lengths are larger at the surface, as in earlier studies of large crystallites $(4,24)$. Particles that lie one or more layer from the perimeter region tend to remain within the cluster and have $C_{6}$ values close to 1 (Fig. 3B). On the other hand, as $N$ falls below $N_{\mathrm{x}}$, a cluster rapidly forms a dense amorphous (liquid) phase. Here, $\left|\psi_{6}\right|^{2}$ av and $C_{6}$ are small throughout the cluster area, which is the signature of a liquid. The liquid phase is thermodynamically unstable, and thus rapidly evaporates into the surrounding gas phase. Although this liquid phase is not found in equilibrium, it has a profound effect on the transition kinetics.

Systems with short-range attraction can also exhibit a second solid phase, which has the same symmetry as the other crystal but lower density (25). This expanded solid is expected to become metastable in the presence of the $3.5 \%$ size polydispersity used here (26). Even when the expanded solid is metastable, however, simulations showed that crystallites nucleate with the density of the (metastable) expanded phase, then contract to the stable-solid density as they grow (27). Correspondingly, we found that our sublimating crystallites dilate slightly as $N$ decreases toward $N_{\mathrm{x}}$. Our data, however, do not directly reveal two solid phases; we therefore focus on the more apparent amorphous phase.

To verify the role of a liquid phase in the sublimation kinetics, we performed simulations in the region of the phase diagram where gas-liquid coexistence is found in equilibrium. In accord with 


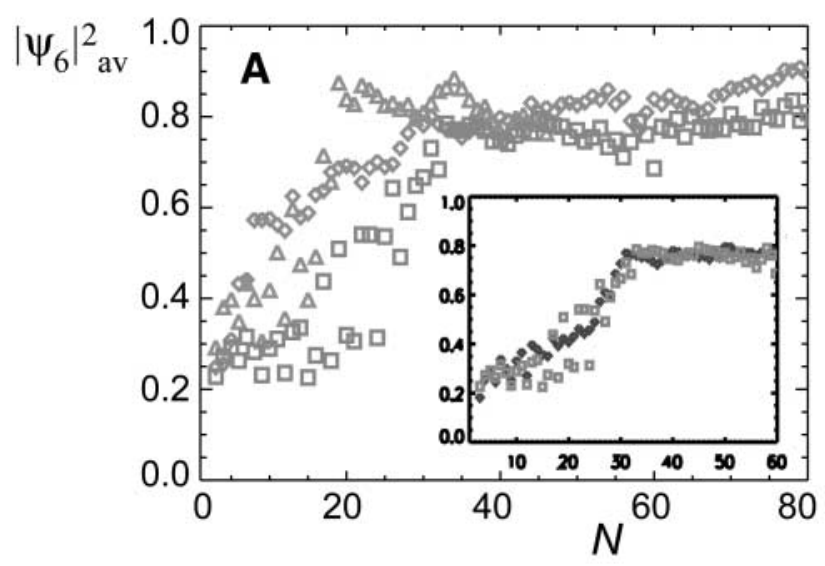

Fig. 3. The sixfold orientational order of clusters. (A) Plot of $\left|\psi_{6}\right|^{2}$ averaged over all clusters of size $N$. These experimental data include all clusters (not just the largest) in all samples studied. Diamonds, SDS at $65^{\circ} \mathrm{C}$ (seven clusters); triangles, $\mathrm{SDS}$ at $30^{\circ} \mathrm{C}$ (two clusters); squares, $\mathrm{C}_{12} \mathrm{E}_{6}$ at $27^{\circ} \mathrm{C}$ (four clusters). (Inset) Data for melting (squares) $\left(T=27^{\circ} \mathrm{C}\right.$; four clusters) and freezing (diamonds) $\left(T=28^{\circ} \mathrm{C}\right.$; two clusters) in the same sample containing colloidal spheres and $C_{12} E_{6}$ micelles. (B) The product $\left(C_{6}\right)$ of each particle's bond-orientational order parameter with that of its neighbors in the same colloidal crystals with $\mathrm{C}_{12} \mathrm{E}_{6}$ at $27^{\circ} \mathrm{C}$. For the $j$ th particle, $C_{6}(j) \equiv\langle I(1 / Z)$ $\left.\left.\Sigma_{\mathrm{k}} \psi_{6}(j) \psi_{6}{ }^{*}(k)\right|^{2}\right\rangle$, where the index $k$ runs over all $Z$ neighbors of $j$. The particles are sorted according to their position: perimeter (asterisks), and first (diamonds) and second (triangles) layers in from the perimeter. The perimeter particles are defined as those with fewer than six bonds; the firstlayer particles have six bonds and are contiguous with the perimeter. The
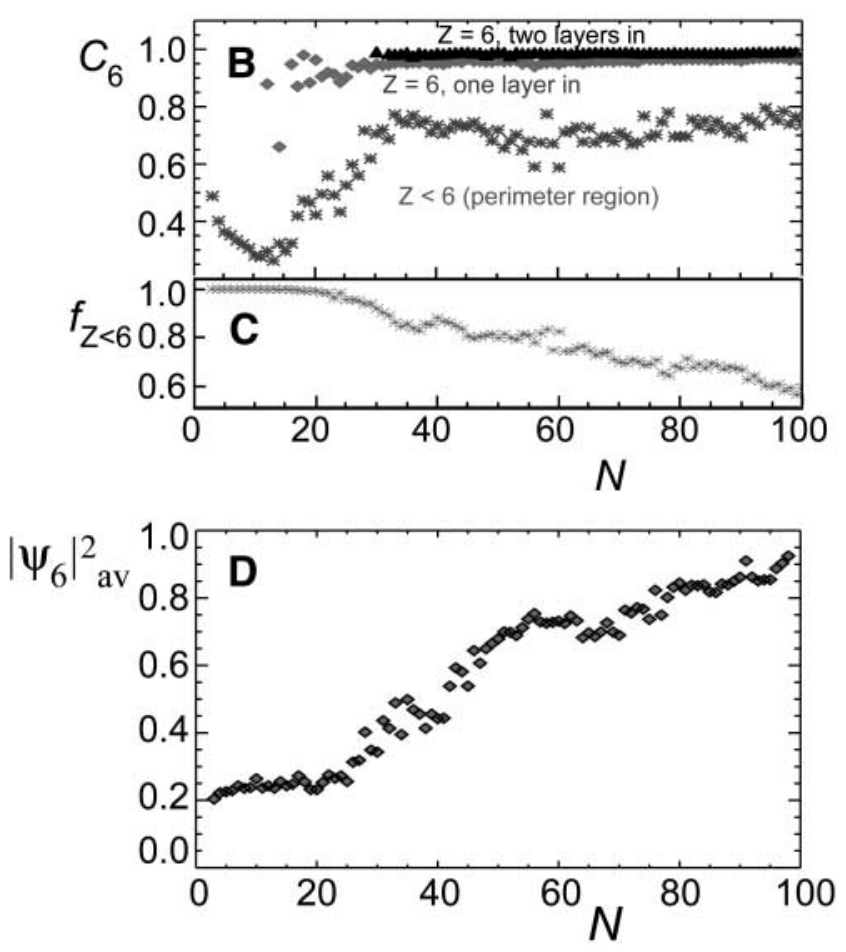

second-layer particles are contiguous with the first layer. (C) The fraction, $f$, of particles that have $Z<6$. (D) $\left|\psi_{6}\right|^{2}$ averaged over all clusters of size $N$ for a computer simulation (averaged over six runs). earlier investigations in two and three dimensions $(4,6,28-30)$, our quasi-two-dimensional simulations reveal only one amorphous phase (gas) in equilibrium when the attraction is of short range. When the range of attraction exceeds approximately $60 \%$ of the diameter, however, we found coexistence of gas and liquid phases in equilibrium (22). Whereas short-range attraction leads to a transient liquid phase, a long-range attraction leads to a stable liquid. For example, when the range of attraction is $80 \%$ of the sphere diameter, a crystallite of $N=100$ rapidly forms a dense liquid droplet, which shrinks to $N 85$ and then persists for at least $1000 R^{2} / D$, where $R$ is the particle radius and $D$ is the diffusion constant. Earlier reports of the melting of ligand-stabilized $\mathrm{CdS}$ nanoparticles in vacuum have also found that the crystalline nanoparticles melt to form stable liquid droplets upon heating (31). Apparently, as our clusters shrink in size, the free energy of the solid phase approaches and then exceeds that of the liquid [which can also be inferred from the evidence that the melting temperature decreases for small crystallites (31-33)]. At this point, forming a liquid from the crystal reduces the energy. Because relatively few bonds need to be broken in order to disrupt the long-range order of the crystallite, this process is plausibly fast. Once the liquid forms, the static shear modulus vanishes; if this liquid is thermodynamically unstable, thermal fluctuations cause rapid evaporation.

Notably, we found a similar dependence of $\left|\psi_{6}\right|^{2}$ and $C_{6}$ on $N$ during freezing (inset of Fig. 3A).
For colloidal crystals in the presence of $\mathrm{C}_{12} \mathrm{E}_{6}$, the sample was subjected to repeated freezing and melting by repeated switching of $T$ from $28^{\circ}$ to $27^{\circ}$ $\mathrm{C}$ (waiting sufficient time between cycles to ensure complete melting and looking in different regions of the sample). During the repeated cycles, crystallites showed statistically indistinguishable $\left|\psi_{6}\right|_{\text {av }}^{2}$ versus $N$. Hence, we conclude that the liquid phase, which is unstable or metastable in this case, is intimately involved in nucleation as well as melting. This conclusion agrees with earlier computer simulations (6), density functional theory $(8,10)$, and experiments $(9)$ in three dimensions.

The results described here were found in crystallites of one to three layers thick, but are likely to be generally applicable to crystallites of arbitrary thickness formed from centrosymmetric, attractive potentials. Short-range attractions might prevail in crystallization and melting of macromolecules such as proteins in membranes or in three dimensional solutions; in these cases, the liquid phase is metastable or unstable and small crystallites would rapidly vanish. Crystallites of atoms with longer-range (Lennard-Jones-like) attraction are common in vacuum-phase film deposition and as nanoparticles on substrates or in solution; in these cases, the liquid phase is stable and small crystallites can form tiny liquid droplets that persist, as indicated by our simulations. In clusters composed of nonspherical particles, other metastable phases with body-centered cubic $(34,35)$ or liquid crystalline symmetry (36) might determine the kinetics. Hence, the observed two- stage sublimation process should be generally relevant to the melting, freezing, and annealing kinetics of small clusters with short-range attraction.

\section{References and Notes}

1. A. Laaksonen, V. Talanquer, D. W. Oxtoby, Annu. Rev. Phys. Chem. 46, 489 (1995).

2. R. W. Cahn, Nature 413, 582 (2001).

3. J. G. Dash, Rev. Mod. Phys. 71, 1737 (1999).

4. V. J. Anderson, H. N. W. Lekkerkerker, Nature 416, 811 (2002).

5. J. W. Cahn, J. Am. Ceram. Soc. 52, 118 (1969).

6. P. R. ten Wolde, D. Frenkel, Science 277, 1975 (1997).

7. R. M. L. Evans, W. C. K. Poon, M. E. Cates, Europhys. Lett. 38, 595 (1997).

8. V. Talanquer, D. W. Oxtoby, J. Chem. Phys. 109, 223 (1998)

9. L. F. Filobelo, O. Galkin, P. G. Vekilov, J. Chem. Phys. 123, 014904 (2005).

10. J. F. Lutsko, G. Nicolis, Phys. Rev. Lett. 96, 046102 (2006).

11. K. Lu, Y. Li, Phys. Rev. Lett. 80, 4474 (1998).

12. Z. H. Jin, P. Gumbsch, K. Lu, E. Ma, Phys. Rev. Lett. 87 055703 (2001)

13. M. Born, J. Chem. Phys. 7, 591 (1939).

14. Y. J. Zhou, X. F. Jin, Phys. Rev. B 71, 224113 (2005).

15. B. I. Halperin, D. R. Nelson, Phys. Rev. Lett. 41, 121 (1978).

16. C. A. Murray, D. H. van Winkle, Phys. Rev. Lett. 58, 1200 (1987).

17. A. H. Marcus, S. A. Rice, Phys. Rev. Lett. 77, 2577 (1996).

18. R. A. Quinn, ]. Goree, Phys. Rev. E Stat. Nonlin. Soft Matter Phys. 64, 051404 (2001)

19. H. H. von Grunberg, P. Keim, K. Zahn, G. Maret, Phys. Rev. Lett. 93, 255703 (2004)

20. R. W. Cahn, Nature 323, 668 (1986)

21. A. E. Larsen, D. G. Grier, Nature 385, 230 (1997).

22. Materials and methods are available as supporting material on Science Online.

23. P. D. Kaplan, J. L. Rouke, A. G. Yodh, D. J. Pine, Phys. Rev. Lett. 72, 582 (1994).

24. A. M. Alsayed, M. F. Islam, J. Zhang, P. J. Collings, A. G. Yodh, Science 309, 1207 (2005). 
25. P. Bolhuis, D. Frenkel, Phys. Rev. Lett. 72, 2211 (1994)

26. P. G. Bolhuis, Ph.D. thesis, University of Utrecht, Netherlands (1996)

27. A. Cacciuto, S. Auer, D. Frenkel, Phys. Rev. Lett. 93, 166105 (2004).

28. H. N. W. Lekkerkerker, W. C. K. Poon, P. N. Pusey, A. Stroobants, P. B. Warren, Europhys. Lett. 20, 559 (1992)

29. S. M. Ilett, A. Orrock, W. C. K. Poon, P. N. Pusey, Phys. Rev. E Stat. Phys. Plasmas Fluids Relat. Interdiscip. Top. 51, 1344 (1995)

30. J. F. Lutsko, G. Nicolis, J. Chem. Phys. 122, 244907 (2005).
31. A. N. Goldstein, C. M. Echer, A. P. Alivisatos, Science 256, 1425 (1992).

32. P. R. Couchman, C. L. Ryan, Philos. Mag. A 37, 369 (1978).

33. M. Wautelet, J. Phys. D 24, 343 (1991).

34. P. R. ten Wolde, M. J. Ruiz-Montero, D. Frenkel, Phys. Rev. Lett. 75, 2714 (1995).

35. Y. C. Shen, D. W. Oxtoby, Phys. Rev. Lett. 77, 3585 (1996).

36. A. M. Alsayed, Z. Dogic, A. G. Yodh, Phys. Rev. Lett. 93, 057801 (2004).

37. We gratefully acknowledge ]. Machta and D. Frenkel for helpful discussions. J.R.S. and A.D.D. acknowledge support from the Research Corporation and NSF grant
DMR-0305395. D.W.B. acknowledges support from NSF grant DMR-0242402, and A.].L. acknowledges support from the NASA Fluid Physics Program (02-OBPR-03-C).

\section{Supporting Online Material}

www.sciencemag.org/cgi/content/full/314/5800/795/DC1

Materials and Methods

Figs. S1 to S4

References

12 April 2006; accepted 29 August 2006

$10.1126 /$ science. 1128649

\section{Pattern Formation and Shape Changes in Self-Oscillating Polymer Gels}

Victor V. Yashin and Anna C. Balazs*

We developed an efficient model for responsive gels that captures large-scale, two-dimensional (2D) deformations and chemical reactions within a swollen polymer network. The 2D calculations allowed us to probe not only volume changes but also changes in sample shape. By focusing on gels undergoing the oscillatory Belousov-Zhabotinsky reaction, we observed traveling waves of local swelling that form a rich variety of dynamic patterns and give rise to distinctive oscillations in the gel's shape. The observed patterns depend critically on the gel's dimensions. The approach provides a useful computational tool for probing the dynamics of chemomechanical processes and uncovering morphological transformations in responsive gels.

$\mathrm{F}$ or a synthetic material to perform sustained mechanical work, it must undergo large-scale, periodic changes in volume or shape. Polymeric gels constitute optimal candidates for use as soft active materials, because controlled modulations of the surrounding solvent can lead to significant, rhythmic expansion and contraction of the gel $(1,2)$. Consequently, such oscillating gels could be used as microactuators (3) for pulsatile drug delivery (4). In addition to their practical utility, oscillating gels provide an ideal medium for investigating nonlinear dynamical phenomena that can arise from a coupling of mechanical and chemical energy. For example, researchers have isolated scenarios where the initial swelling and deswelling of a chemoresponsive gel exerts feedback on a nonoscillatory chemical reaction and thereby drives the entire system into a regular, oscillatory regime (4-8). Theoretical models for oscillating gels (2, 7-9) have yielded substantial insight into mechanisms that can produce periodic pulsations. Prior calculations (2, 7-9), however, were carried out in one dimension (e.g., the sample was assumed to be spherically symmetric), and thus only volumetric changes of the sample could be probed. To capture shape changes, models must describe the gel deformation in at least two dimensions. By encompassing additional degrees of freedom,

Chemical Engineering Department, University of Pittsburgh, Pittsburgh, PA 15261, USA.

*To whom correspondence should be addressed. E-mail: balazs1@engr.pitt.edu

2D (or 3D) models can enhance our fundamental understanding of the interplay between the finite deformations of a responsive medium and the nonlinear chemical dynamics and open up the possibility of uncovering new morphological transitions (7).
Fig. 1. Temporal evolution of $\phi$ and $v$ in a 30-by-30 square gel. (A) Location of horizontal (a-b) and vertical (c-d) cross sections through the sample. (B) Nonresponsive gel $\left(\chi^{*}=0\right)$. (C) Responsive gel $\left(\chi^{*}=0.105\right)$. Rhythmic variation of gel size is clearly seen. Simulations were performed with (9): $\phi_{0}=0.139, \quad c_{0} v_{0}=$ $1.3 \times 10^{-3}, \chi(\phi)=0.338+$ $0.518 \phi, \quad \lambda_{\perp}=1.1, \varepsilon=$ $0.354, f=0.7$, and $q=$ $9.52 \times 10^{-4}$.
We developed an approach for simulating chemoresponsive gels that can exhibit not only large variations in volume but also alterations in shape. Through this approach, we modeled oscillating gels undergoing the BelousovZhabotinsky (BZ) reaction (10-12) and showed that the ensuing $2 \mathrm{D}$ pattern formation depends on the dimensions of the sample. BZ gels are unique because the polymer network can expand and contract periodically without external stimuli $(11,12)$. This autonomous, self-oscillatory behavior is due to a ruthenium catalyst, which is covalently bonded to the polymers $(11,12)$. The $\mathrm{BZ}$ reaction generates a periodic oxidation and reduction of the anchored metal ion, and these chemical oscillations induce the rhythmic swelling-deswelling in the gel. By probing morphological changes in BZ gels, we can establish design criteria for creating autonomous smallscale devices, which perform sustained work until the reagents in the host solution are consumed and can be simply refueled by replenishing these solutes.

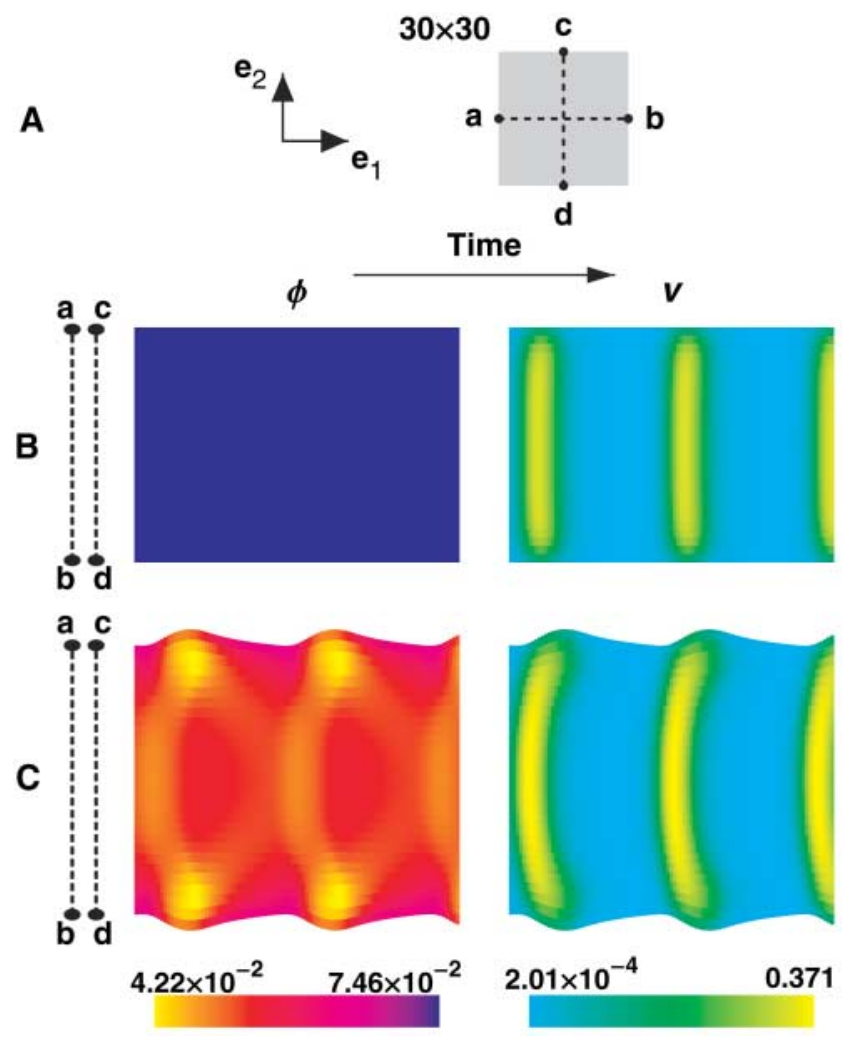

